\title{
Three-Dimensional Imaging of Atomic Four-Body Processes
}

M. Schulz ${ }^{1,2}$, R. Moshammer ${ }^{2}$, D. Fischer ${ }^{1}$, H. Kollmus ${ }^{1}$, D.H. Madison ${ }^{2}$, S. Jones ${ }^{2}$, and J. Ullrich ${ }^{1}$

\author{
${ }^{1}$ Max-Planck Institut für Kernphysik, Saupfercheckweg 1, D-69117 Heidelberg, Germany \\ ${ }^{2}$ University of Missouri-Rolla, Physics Department and Laboratory for Atomic, Molecular, \\ and Optical Research, Rolla, Missouri 65409, USA
}

Understanding processes occurring in nature requires obtaining a solid concept about the "fundamental" forces acting between pairs of elementary particles. We then try to understand the temporal and spatial evolution of many mutually interacting particles under the influence of these forces. This latter step, known as the few-body problem, remains one of the unsolved, fundamentally important problems in physics. Atomic collisions represent a particularly suitable testground to study the few-body problem. For single ionization of He by charged particle impact kinematically complete experiments have been performed ${ }^{1-6}$ since the pioneering work of Ehrhardt et al. ${ }^{7}$ It was thought, that a profound knowledge of the basic features observed in fully differential measurements had emerged, at least for large collision energies. ${ }^{8-14}$ These conclusions are, however, almost exclusively based on studies which were performed for restricted electron-emission geometries. ${ }^{1-3}$ Here, we present measured three-dimensional images of the complete electron emission pattern for single ionization of helium by $100 \mathrm{MeV} / \mathrm{amu} \mathrm{C}^{6+}$ impact (a four-body system). In the data 
we observe unexpected features which so far were not predicted by any published theoretical model. We propose a higher-order ionization mechanism involving the projectile - target nucleus interaction to explain these features.

The experimental set-up ${ }^{15}$ is schematically shown in Fig. 1. 100 MeV/amu $\mathrm{C}^{6+}$ projectiles collided with cold $(<1 \mathrm{~K}) \mathrm{He}$ gas target atoms from a supersonic jet. The recoiling target ions and the ionized electrons were extracted by a weak electric field and detected by two-dimensional position sensitive channel plate detectors. A uniform magnetic field of 12 Gauss parallel to the electric field forced the electrons into cyclotron motion (green spiral line) and thereby confined their motion in the plane perpendicular to the extraction field (transverse plane) so that all electrons with a transverse momentum of less than 2 atomic units (a.u.) hit the detector. Both particles were measured in coincidence with the projectiles which did not change charge state. The momentum components of the recoil ion and the electron in the direction of the extraction field (longitudinal components) were determined from the time of flight of each particle from the collision region to the respective detector. The transverse momenta were obtained from the position information provided by the detectors for a fixed time of flight.

In the top of Fig. 2, the measured three-dimensional fully differential cross sections (FDCS) are shown as a function of the azimuthal $\left(\varphi_{\mathrm{e}}\right)$ and polar $\left(\theta_{\mathrm{e}}\right)$ electron angles. Although the data are only shown for a momentum transfer (defined as the difference between the scattered and initial projectile momentum) $q=0.75$ a.u. and an ionized electron energy $E_{\mathrm{e}}=6.5 \mathrm{eV}$, all other final electron energies $\mathrm{E}_{\mathrm{e}}<50 \mathrm{eV}$ and a large fraction of all possible momentum transfers are recorded simultaneously but not discussed in this letter. 
The initial projectile direction is along the z-axis and $\mathbf{q}$ is pointing nearly in the negative $\mathrm{x}$ direction. $\varphi_{\mathrm{e}}$ is measured in the xy-plane relative to the negative momentum transfer direction, i.e. $\varphi_{\mathrm{e}}=0^{\circ}$ and $180^{\circ}$ correspond to the scattering plane (i.e. the plane spanned by the initial projectile momentum and the momentum transfer vectors). $\theta_{\mathrm{e}}$ is the angle of the electron momentum vector relative to the $\mathrm{z}$-axis.

The characteristic double lobe structure with peaks at $\left(\theta_{\mathrm{e}}=90^{\circ}, \varphi_{\mathrm{e}}=0^{\circ}\right)$ and $\left(\theta_{\mathrm{e}}=90^{\circ}\right.$, $\varphi_{\mathrm{e}}=180^{\circ}$ ) well-known from high-energy electron-impact studies in the scattering plane $\mathrm{e}^{1-3}$ is clearly observable. The $\left(\theta_{\mathrm{e}}=90^{\circ}, \varphi_{\mathrm{e}}=180^{\circ}\right)$ - maximum (dubbed the binary peak), which occurs in the direction of $\mathbf{q}$, is relatively easy to understand. It is due to collisions which are dominated by a binary interaction between the projectile and the electron, i.e. the recoil ion remains essentially passive. Momentum conservation then demands the electron to be emitted approximately in the direction of $\mathbf{q}$. The $\left(\theta_{\mathrm{e}}=90^{\circ}, \varphi_{\mathrm{e}}=0^{\circ}\right)$ - lobe, called the recoil peak, has been interpreted as a double scattering process: ${ }^{16}$ the electron, initially emitted approximately in the direction of $\mathbf{q}$, on its path out of the atom elastically backscatters from the recoil ion, which picks up most of the momentum transferred from the projectile.

In our current understanding, the ionization cross sections at perturbations as small as studied in this work should be essentially identical for electron and ion impact. Furthermore, the results of various theoretical approaches converge to each other with decreasing perturbation. For our collision system, it is therefore not critical which model is used for comparison with the data in order to identify potential deviations from our current understanding. We chose a continuum distorted wave approach with Hartree-Fock 
wavefunctions for the active electron $(\mathrm{CDW}-\mathrm{HF})$ because it is the currently most powerful model available for ion impact ionization.

The bottom part of Fig. 2 shows our theoretical FDCS for the same conditions as the experimental data. This CDW-HF calculation is based on the work by Belkic ${ }^{10}$ and Crothers. ${ }^{11}$ In our model, ${ }^{12,13}$ the initial state of the collision is represented by a plane wave for the projectile and a Hartree-Fock wavefunction for the He ground state of the electrons. The final state is given by a product of three terms - one for each two-particle subsystem of the final three-particle state. A Coulomb wave is used for the projectile - recoil ion subsystem (i.e. a $\mathrm{C}^{6+}$ ion in the field of a $\mathrm{He}^{+}$ion) and a Coulomb interaction is used for the projectile - electron subsystem. A numerical solution of the Schrödinger equation for an electron in the field of a $\mathrm{He}^{+}$ion represented by a static Hartree-Fock potential is employed for the electron - recoil ion subsystem. Although this model represents a state-of-the-art calculation for single ionization by ion impact, significant qualitative differences to the experimental data are apparent. In particular, the calculation is nearly cylindrically symmetric about the $\mathrm{x}$-axis (momentum transfer direction) while the data are much broader in the xy-plane than in the xz-plane.

If the ionization process is dominated by a single projectile-target interaction, the symmetry properties of the initial $1 \mathrm{~s}^{2}{ }^{1} \mathrm{~S}$ state lead to cylindrical symmetry about $\mathbf{q}$ in the FDCS. Any deviation from that symmetry is due to higher order contributions in the interaction between the projectile and the target. The fact that our calculation is nearly cylindrically symmetric shows that in the theory higher-order processes are insignificant. In contrast, the pronounced deviation in the experimental data from that symmetry indicates that higher-order contributions are actually surprisingly important. 
To study the discrepancies between the data and the calculation in more detail, we show in Fig. 3 the experimental (closed symbols) and theoretical (solid curves) FDCS for two different cuts through the 3-dimensional emission patterns of Fig. 1. The first cut selects the scattering plane (indicated by the blue line in Fig. 1) and the second cut the plane perpendicular to $\mathbf{q}$ (red line in Fig. 1). For each selected plane, the FDCS are plotted as a function of the electron emission angle $\theta_{\mathrm{e}}$ measured relative to $\mathbf{p}_{\mathbf{o}}$. For the scattering plane, $\theta_{\mathrm{e}}=90^{\circ}$ corresponds to the direction of $\mathbf{q}$ and $\theta_{\mathrm{e}}=270^{\circ}$ to $-\mathbf{q}$ (note that here $\theta_{\mathrm{e}}$ is a plane polar coordinate as opposed to the spherical polar angle used in Fig. 1).

In the scattering plane, the calculation is in good agreement with the data except for some quantitative differences in the recoil peak. It should be noted that both the data and the calculation are absolute, i.e. there is no adjustment in the absolute magnitude. Such a good reproduction of the data by theory in the scattering plane is quite typical for single ionization of $\mathrm{He}$ by high-energy electron impact as well, even when compared to less sophisticated models such as the first Born approximation. ${ }^{17}$ In that sense, our results are consistent with high-energy electron-impact data. In the perpendicular plane, in contrast, dramatic discrepancies between experiment and theory are quite obvious. Here, the theory is essentially isotropic and does not show the peak structures perpendicular to the beam direction, which are still fairly pronounced in the data. Such an isotropic emission pattern for the perpendicular plane is again characteristic of a first-order ionization process. Therefore, the structures observed in the data further support our conclusion above concerning surprisingly strong contributions from higher-order processes. 
An important hint for the cause of the discrepancies between our experimental and theoretical results is contained in the perpendicular plane FDCS measured for $2 \mathrm{MeV} / \mathrm{amu}$ $\mathrm{C}^{6+}+\mathrm{He}$ collisions. These cross sections are shown in Fig. 3 for $\mathrm{q}=0.7$ a.u. and $\mathrm{E}_{\mathrm{e}}=4 \mathrm{eV}$ (open symbols) and for $\mathrm{q}=1.5$ a.u. and $\mathrm{E}_{\mathrm{e}}=1 \mathrm{eV}$ (closed symbols). In the former case, the maxima near $90^{\circ}$ and $270^{\circ}$ observed for the larger projectile energy are completely absent. In the latter case the electron momentum (0.27 a.u.) is very small compared to $\mathrm{q}$ so that here the momentum exchange in the collision occurs predominantly between the projectile and the recoil ion. Under these conditions we observe maxima near $90^{\circ}$ and $270^{\circ}$, as we do for the large projectile energy.

This comparison of the data in Fig. 4 provides a strong indication that the peak structures perpendicular to the projectile beam observed in the perpendicular plane are due to a higher-order process involving the projectile - target nucleus interaction. We propose the following two-step mechanism to explain the data: in the first step, the electron is ejected due to a binary projectile-electron interaction approximately in the direction of the momentum q' transferred in this initial interaction. The projectile then elastically scatters from the target nucleus. In this second step an additional momentum q" is transferred, which may be pointing out of the initial scattering plane $\left(\mathbf{p}_{\mathbf{0}}, \mathbf{q}^{\prime}\right)$. The total momentum transfer $\mathbf{q}=\mathbf{q}^{\prime}+\mathbf{q}$ ' measured in the experiment is then rotated about the projectile beam axis relative to q'. If this rotation, which does not affect the direction of the electron momentum, is $90^{\circ}$, the electron ends up in the perpendicular plane $\left(\mathbf{p}_{\mathbf{o}}, \mathbf{q}^{\mathbf{9}}\right)$ which started off as the scattering plane after the initial projectile-electron interaction. Since the electron does not take part in the second step, its polar angular distribution remains unchanged leading to a peak at the same angle as in the scattering plane. 
This mechanism has a significant impact on basic features characteristic to ionization which were previously thought to be understood. What appears to be a recoil peak in the scattering plane emerges as a "recoil ring" in the measured 3-dimensional electron emission pattern which is nearly isotropic with respect to rotation about the projectile beam axis. This behavior, not reproduced by theory, can qualitatively also be explained by the mechanism suggested above: if the projectile - target nucleus interaction leads to a rotation of $\mathbf{q}$ about the projectile beam axis by $180^{\circ}$ then the ionized electron is emitted in the direction of -q (i.e. it looks like a "recoil peak electron") although it was not back-scattered by the target nucleus. This could be an important contributor to the recoil peak which so far has not been discussed in the literature. Such contributions would also explain small, but systematic underestimations of the height of the recoil peak by theory both for electron and ion impact. ${ }^{1,13,17}$

This process represents a potentially important few-body effect involving all collision products. In principle, it is contained in the final state wavefunction in our calculation. Furthermore, it should be pointed out that, for the sake of a graphic explanation, our description of this mechanism represents a simplification. The CDW-HF model treats this process more realistically in that here the projectile - electron and projectile - target nucleus interaction do not occur sequentially, but simultaneously instead. However, the final state wavefunction is only asymptotically exact when at least one of the collision products is at a large distance from the other two particles and its accuracy is not known when all particles are close together. On the other hand, this process is expected to be important exactly when all collision products are still close together and may therefore be significantly underestimated by the calculation. 
Finally, we want to allude to a promising theoretical development. Very recently, the CDW-approach has been generalized to include non-zero magnetic sub-states in the final state wavefunction. ${ }^{14}$ Such sub-states would be expected to contribute to the cross sections out of the scattering plane and therefore offer an alternative or complementary explanation to the one proposed above. However, numeric calculations on fully differential cross sections with this method are not available yet.

In conclusion, existing theoretical approaches should be tested relative to their capability of reproducing measured 3-dimensional electron emission patterns. Such tests may reveal that qualitatively new theoretical concepts need to be developed in order to achieve a better understanding of ionization processes even at large projectile energies. The data presented here provide an experimental benchmark for such efforts. Obtaining a satisfactory description of atomic few-body processes, in turn, is an important step towards a better understanding of the general few-body problem.

Correspondence and requests for materials to Michael Schulz, schulz@umr.edu

\section{References}

[1] Ehrhardt H., Jung K., Knoth G., and Schlemmer P. Differential cross sections of direct single electron impact ionization, Z. Phys. D1, 3 (1986) and references therein

[2] Stefani G., Avaldi L., and Camilloni R. On the relevance of the final ionic state to the (e, 2e), TDCS J. Phys. B23, L227 (1990)

[3] Lahmam-Bennani A. Recent developments and new trends in (e,2e) and (e,3e) studies, J. Phys. B24, 2401 (1991) 
[4] Murray A.J., Woolf M.B.J., and Read F.H. Results from symmetric and nonsymmetric energy sharing (e, 2e) experiments in the perpendicular plane, J. Phys. B25, $3021(1992)$

[5] Röder J., Ehrhardt H., Bray I., Fursa D.V., McCarthy I.E. Absolute triple differential cross section for electron-impact ionization of helium at $40 \mathrm{eV}$, J. Phys. B29, 2103 (1996)

[6] Schulz M. et al. Triply differential single ionization cross sections in coplanar and noncoplanar geometry for fast heavy ion-atom collisions, J. Phys. B34, L305 (2001)

[7] Ehrhardt H., Schulz M., Tekaat T., and Willmann K. Ionization of helium: angular correlation of the scattered and ejected electrons, Phys. Rev. Lett. 22, 89 (1969)

[8] Bray I. and Stelbovics A.T. Explicit demonstration of the convergence of the closecoupling method for a Coulomb three-body problem, Phys. Rev. Lett. 69, 53 (1992)

[9] Rescigno T.N., Baertschy M., Isaacs W.A., and McCurdy C.W. Collisional breakup in a quantum system of three charged particles, Science 286, 2474 (1999)

[10] Belkic Dz., A quantum theory of ionisation in fast collisions between ions and atomic systems, J. Phys. B11, 3529 (1978)

[11] Crothers D.S.F. and McCann J.F., Ionisation of atoms by ion impact, J. Phys. B16, $3229(1983)$

[12] Jones S. and Madison D.H. Evidence of initial-state two-center effects for (e, 2e) reactions, Phys. Rev. Lett. 81, 2886 (1998)

[13] Madison D., Schulz M., Jones S., Foster M., Moshammer R. and Ullrich J. Comparison of theoretical and absolute experimental fully differential cross sections for ion-atom impact ionization, J. Phys. B15, 3297 (2002) 
[14] Crothers D.S.F., McSherry D.M., O'Rourke S.F.C., Shah M.B., McGrath C., and Gilbody H.B., Magnetically Quantized Continuum Distorted Waves, Phys. Rev. Lett. $88,053201(2002)$

[15] Ullrich J. et al. Recoil-ion momentum spectroscopy, J. Phys. B30, 2917 (1997)

[16] Whelan C.T., Allan R.J., Walters H.R.J., and Zhang X. (e,2e), effecctive charges, distorted waves and all that!, (e,2e), in Whelan C.T., Walters H.R.J., LahmamBennani A., and Ehrhardt H. (editors), (e,2e) \& related processes, Kluwer, Dordrecht, Series C: Mathematical and Physical Sciences - Vol. 414, 1-32 (1993)

[17] Marchalant P., Whelan C.T., and Walters H.R.J. Second-order effects in (e, 2e) excitation-ionization of helium to $\mathrm{He}^{+}(\mathrm{n}=2)$, J. Phys. B31, 1141 (1998)

\section{Acknowledgements}

The support of the Deutsche Forschungsgemeinschaft within the the Leibniz-program, by GSI, the European Union, the NSF and by CIRIL (GANIL at Caen) as well as fruitful discussions with Drs. R.E. Olson and J. Fiol are gratefully acknowledged. We would like to thank Drs. W. Schmitt, R. Mann, R. Dörner, T. Weber, Kh. Khayyat, A. Cassimi, L. Adoui, J.P. Grandin and staff members of the CIRIL for their indispensable help performing the experiment at the GANIL.

\section{Figure Captions}

Fig. 1: Schematic experimental set-up for three-dimensional imaging of atomic processes. The projectile-ion beam (purple arrow) is crossed with an atomic $\mathrm{He}$ beam from a supersonic gas jet (black arrow). The ionized electrons and the recoiling target ions are 
extracted by a weak electric field (blue arrow) and detected by two-dimensional positionsensitive detectors. A pair of Helmholtz coils generates a uniform magnetic field (yellow arrow) which forces the electrons into cyclotron trajectories (green spiral line) and guides them onto the detector. Two electronic clocks are used to measure the time of flight of the electrons and the recoil ions from the collision region to the respective detector.

Fig. 2: Three-dimensional images of electrons ejected in $100 \mathrm{MeV} / \mathrm{amu} \mathrm{C}^{6+}+\mathrm{He}$ collisions. Experimental data (top) and theoretical results (bottom) are shown for an electron energy of $\mathrm{E}_{\mathrm{e}}=6.5 \mathrm{eV}$ and a momentum transfer of $\mathrm{q}=0.75$ a.u. The scattering plane and the perpendicular plane are indicated by the blue and red lines, respectively.

Fig. 3: Triply differential single ionization cross sections for selected electron emission planes. Experimental (solid circles) and theoretical (solid curves) results are shown for the scattering plane (left, blue line in Fig. 1) and for the perpendicular plane (right, red line in Fig. 1).

Fig. 4: Triply differential single ionization cross sections for the perpendicular plane for 2 MeV/amu $\mathrm{C}^{6+}+\mathrm{He}$ collisions. Open symbols: $\mathrm{E}_{\mathrm{e}}=4 \mathrm{eV}, \mathrm{q}=0.7$ a.u.; closed symbols: $\mathrm{E}_{\mathrm{e}}$ $=1 \mathrm{eV}, \mathrm{q}=1.5$ a.u. 
Electron

detector

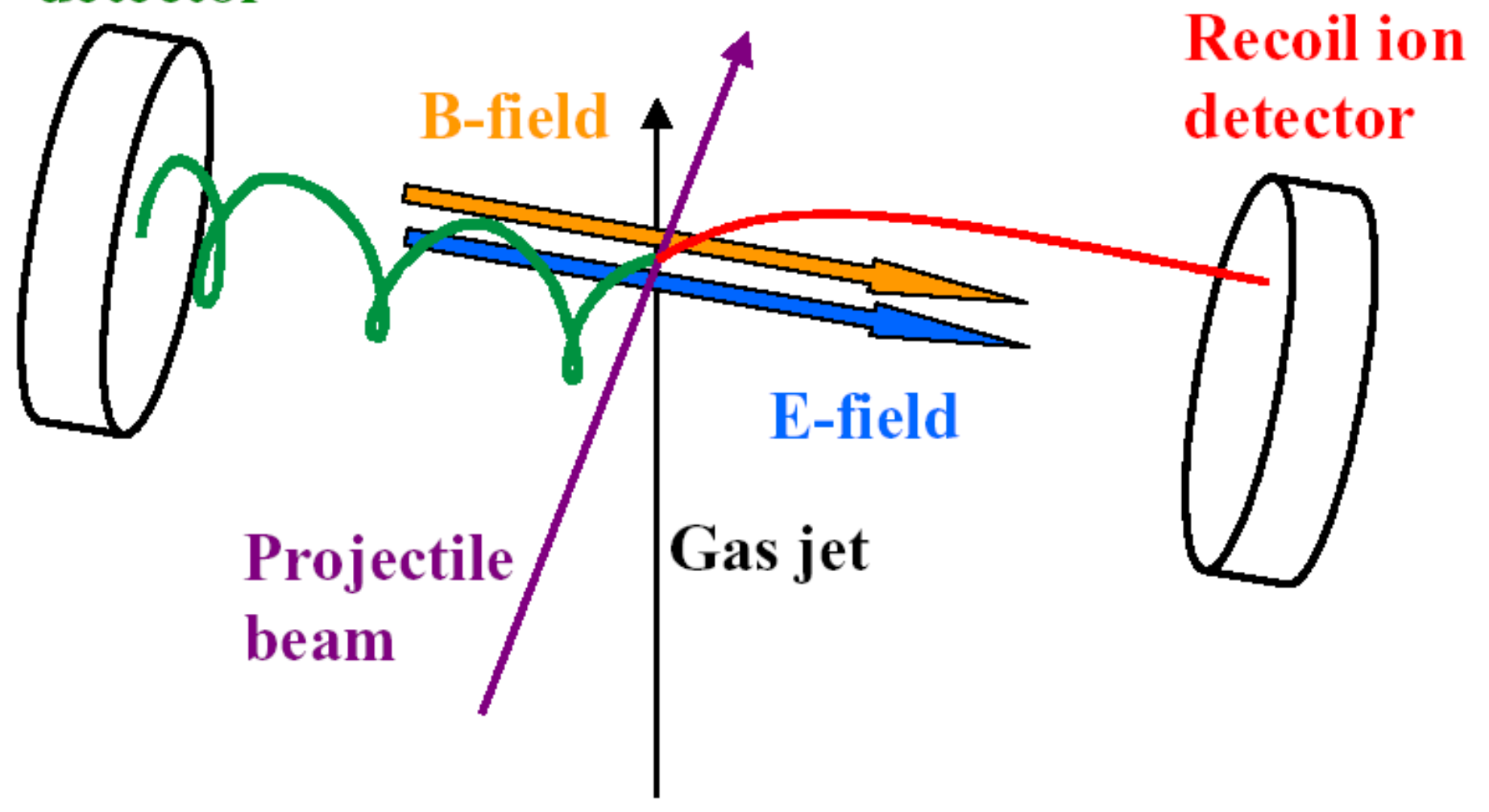

FIGURE. 1 

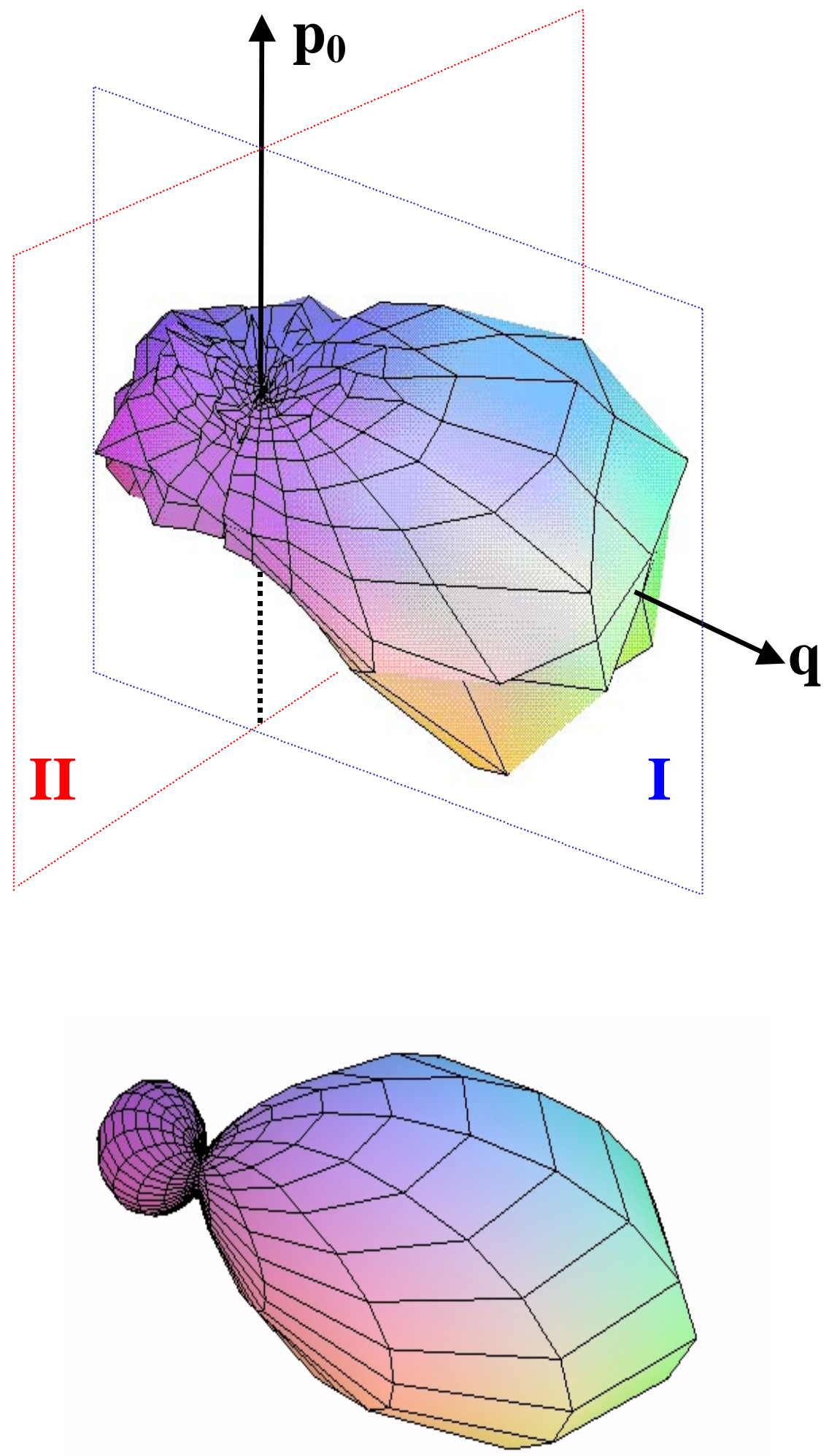

FIGURE. 2 

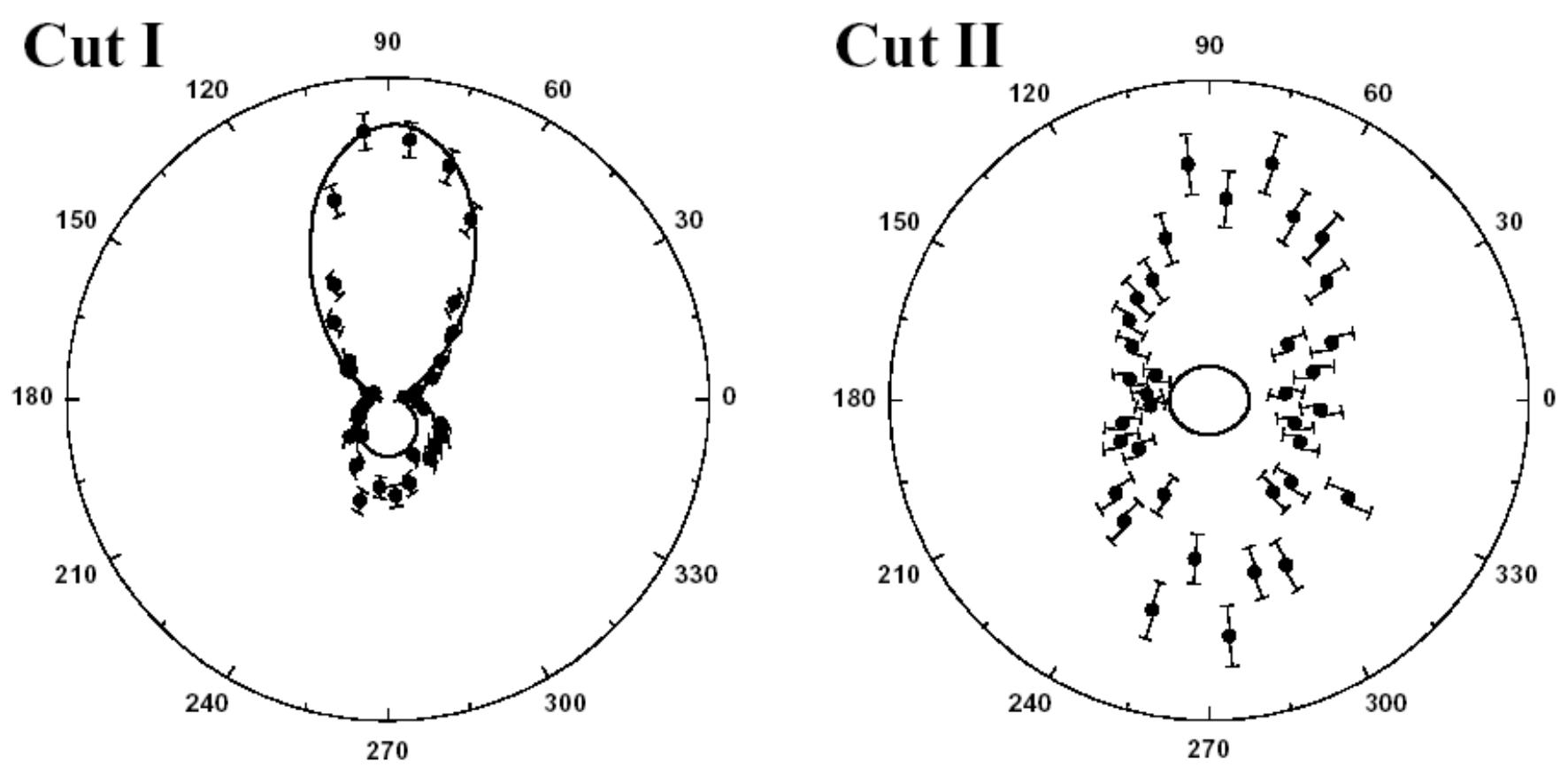

FIGURE. 3 


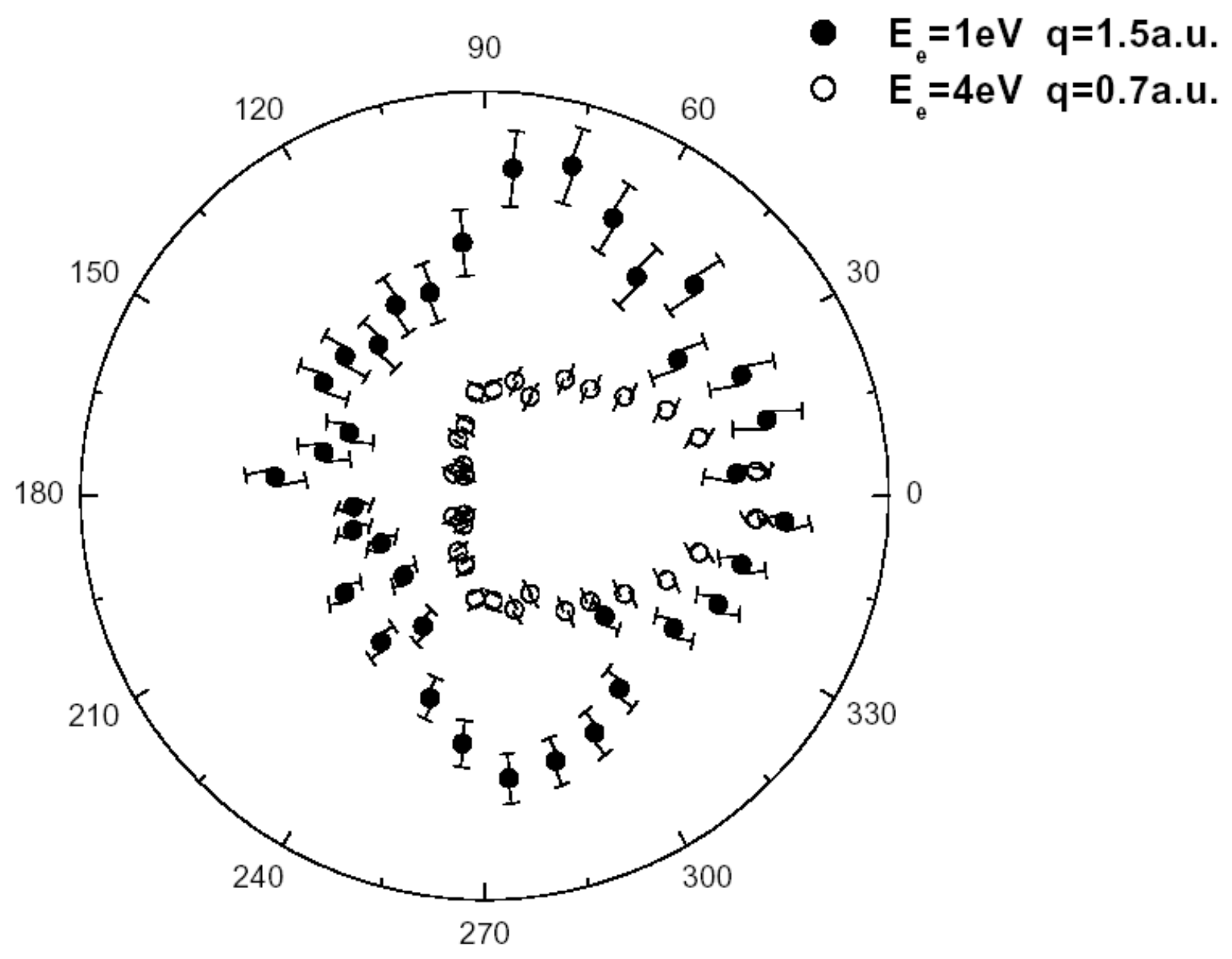

FIGURE. 4 
